\title{
Strong Coupling of Surface Plasmon Polaritons in Monolayer Graphene Sheet Arrays
}

\author{
Bing Wang, ${ }^{1, *}$ Xiang Zhang, ${ }^{2}$ Francisco J. García-Vidal, ${ }^{3}$ Xiaocong Yuan, ${ }^{4}$ and Jinghua Teng ${ }^{1, \dagger}$ \\ ${ }^{1}$ Institute of Materials Research and Engineering, Agency for Science, Technology, \\ and Research (A*STAR), 3 Research Link, Singapore 117602 \\ ${ }^{2}$ Department of Physics, Wuhan University, Wuhan 430072, China \\ ${ }^{3}$ Departamento de Física Teórica de la Materia Condensada, Universidad Autónoma de Madrid, E-28049 Madrid, Spain \\ ${ }^{4}$ Institute of Modern Optics, Key Laboratory of Optical Information Science and Technology,
}

Nankai University, Tianjin 300071, China

(Received 27 March 2012; published 16 August 2012)

\begin{abstract}
Here we investigate theoretically and numerically the coupling between surface plasmon polaritons (SPPs) in monolayer graphene sheet arrays that have a period much smaller than the wavelength. We show that when the collective SPP is excited with an out-of-phase illumination, the beam tends to propagate toward the opposite direction of the Bloch momentum, reflecting a negative coupling between the constituent SPPs. In contrast, for in-phase illumination, the incident beam is split into two collective SPPs that are highly collimated and display low propagation loss. Moreover, the coupling between the individual SPPs results in a reduction of the modal wavelength of the SPP in comparison with that of a single graphene sheet.
\end{abstract}

PACS numbers: 42.82.Et, 42.25.Fx, 42.79.Fm, 73.20.Mf

Graphene, an allotrope of carbon consisting of $s p^{2}$ bonded carbon atoms arranged in one atom thick honeycomb lattice, has attracted extensive attention since the practical production of stable graphene in 2004 [1]. A great diversity of electronic and optical effects have been found in graphene such as integer and fractional quantum Hall effect at room temperature, tunable band gap, ballistic electronic propagation, optical saturable absorption and luminescence [2-4]. The optical response of graphene is characterized by its surface conductivity which greatly relates to its chemical potential (Fermi energy). Graphene manifests strong absorption of light in the near-infrared and visible range [5]. At lower frequencies such as $\mathrm{THz}$ and far-infrared range, the intraband transition of electrons dominates and graphene behaves like a metal. The transverse magnetic (TM) polarized surface plasmon polaritons (SPPs) could therefore be supported by graphene. SPPs in graphene possess unique features as compared with metals, such as huge modal index, relatively low loss, and flexible tunability by electric field, magnetic field, and gate voltage [6]. These features make graphene a promising material for SPP-based optical nanodevice applications.

So far, the study on graphene plasmonics mostly focused on SPPs in monolayer graphene, graphene ribbons, and double-layer graphene sheets [6-10]. The excitation of SPPs in graphene is theoretically proposed by using nanoemitters in graphene sheets [11,12]. Experimental observation of SPPs in graphene has also been reported by using electron spectroscopy [9] and near-field microscopy $[13,14]$. As a fundamental issue, the weak coupling of SPPs in between double-layer graphene sheets was recently investigated [15]. The coupling of SPPs in a periodic multilayer graphene system, which is physically different from that in double-layer graphene sheets, is also an important topic but has not been explored yet.

In this Letter, we propose a monolayer graphene sheet array (MGSA) composed of periodically stacked monolayer graphene sheets with identical interlayer space. The configuration follows the conventional dielectric and metallic waveguide arrays [16-18] that have shown interesting properties such as diffraction-free beam propagation and negative refraction. We will use such a configuration to study the coupling of graphene sheet arrays as a discrete optical system and explore new effects from the tunable properties and ultrathin thickness of graphene.

A diagram of the MGSA is shown in Fig. 1(a). The graphene sheets with a period of $d$ are embedded in the dielectric medium with a relative permittivity denoted by $\varepsilon_{d}$. The surface conductivity of graphene, $\sigma_{g}$, is governed by the Kubo formula [19] including the interband and intraband transition contributions. It is related to temperature $T$, chemical potential $\mu_{c}$, momentum relaxation time $\tau$, and photon frequency $\omega$. In the $\mathrm{THz}$ and far-infrared range, the intraband transition contribution dominates [8] and the surface conductivity simplifies to $\sigma_{g}=\frac{i e^{2} \mu_{c}}{\pi \hbar^{2}\left(\omega+i \tau^{-1}\right)}$ on condition that $\mu_{c} \gg k_{B} T$, where $e$ is the electron charge and $k_{B}$ Boltzmann's constant. In this study, the excitation wavelength of $\lambda=10 \mu \mathrm{m}(\hbar \omega=0.124 \mathrm{eV})$ in air is initially considered and the chemical potential of graphene is assumed to be $\mu_{c}=0.15 \mathrm{eV}$. The momentum relaxation time is chosen as $\tau=0.5 \mathrm{ps}$, corresponding to a mean free path up to $500 \mathrm{~nm}$ at room temperature $(T=300 \mathrm{~K})$ that coincides with the experiment [20,21].

We start from investigating the dispersion relation of SPPs in the MGSA. Considering any two neighboring periods of the array, for example, the region in $-d<x<d$ as 
shown in Fig. 1(a), the magnetic field in between adjacent graphene sheets for TM polarized SPPs can be written as

$H_{y}=\left\{\begin{array}{ll}A^{+} \exp [-\kappa(x+d)]+A^{-} \exp (\kappa x) & -d<x<0 \\ B^{+} \exp (-\kappa x)+B^{-} \exp [\kappa(x-d)] & 0<x<d\end{array}\right.$,

where $\kappa=\sqrt{k_{z}^{2}-\varepsilon_{d} k_{0}^{2}}$ with $k_{z}$ the wave vector of SPPs in the $z$ direction and $k_{0}=2 \pi / \lambda$ being the wave vector in air, $A^{ \pm}$and $B^{ \pm}$represent the amplitudes of SPPs modes damping toward $\pm x$ directions in between adjacent graphene sheets. According to the Bloch theorem, we have $B^{ \pm}=A^{ \pm} \exp \left(i k_{x} d\right)$, where $k_{x}$ is the Bloch wave vector along the $x$ direction. According to Maxwell's equations, the tangential electric field $E_{z}=\frac{i \eta_{0}}{k_{0} \varepsilon_{d}} \frac{\partial H_{y}}{\partial x} \propto \frac{i \eta_{0} \kappa}{k_{0} \varepsilon_{d}}$, where $\eta_{0}$ is the impedance of air. Considering the boundary conditions [8] $E_{z}^{+}=E_{z}^{-}$and $H_{y}^{+}-H_{y}^{-}=\sigma_{g} E_{z}$ at $x=0$ with $E_{z}^{ \pm}$and $H_{y}^{ \pm}$being the fields at the two sides of graphene, we can get the dispersion relation

$$
\cos (\varphi)=\cosh (\kappa d)-\frac{\kappa \xi}{2} \sinh (\kappa d),
$$

where $\xi=\eta_{0} \sigma_{g} /\left(i \varepsilon_{d} k_{0}\right)$ and $\varphi=k_{x} d$ being the Bloch momentum [22,23]. Considering that $\omega \gg \tau^{-1}, \xi$ is nearly a real number and we find $\xi \approx 46 \mathrm{~nm}$ at $\lambda=10 \mu \mathrm{m}$ and $\mu_{c}=0.15 \mathrm{eV}$. In case $d$ is large enough, $k_{z}$ approaches the wave vector of SPPs in monolayer graphene $k_{\mathrm{SPP}}=k_{0} \sqrt{\varepsilon_{d}+\left(\frac{2}{k_{0} \xi}\right)^{2}}$. Thus the modal wavelength of SPPs in monolayer graphene is given by $\lambda_{\mathrm{SPP}}=2 \pi /$ $\operatorname{Re}\left(k_{\mathrm{SPP}}\right) \approx \pi \xi$. At a given frequency, Eq. (2) develops into the diffraction relation $k_{z}(\varphi)$. The $k_{z}(\varphi)$ curves in the

(a)
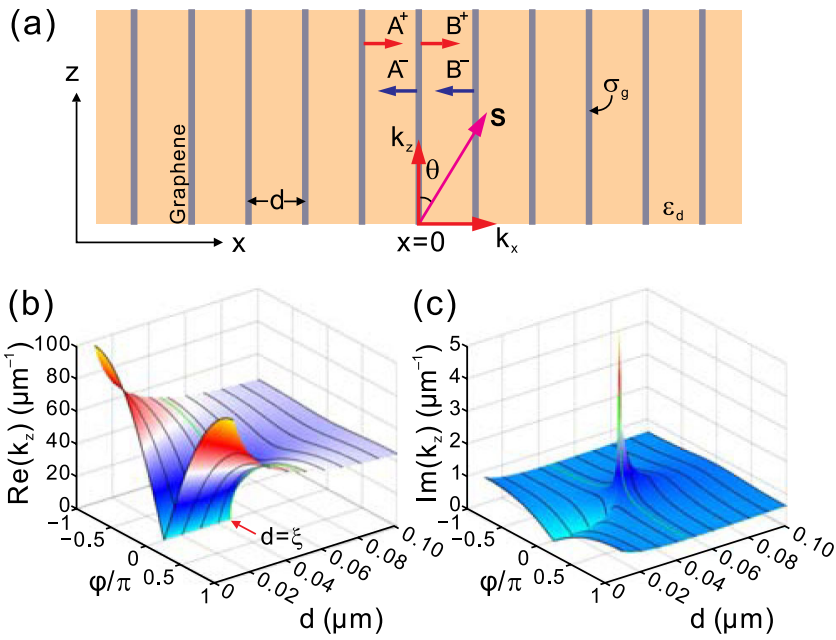

FIG. 1 (color online). Schematic of MGSAs and diffraction relation of SPPs. (a) Schematic of MGSAs. (b) Diffraction relation of SPPs in the MGSA as the period $d$ is varying. (c) $\operatorname{Im}\left(k_{z}\right)$ as a function of $\varphi$ and $d$, where $\varphi=k_{x} d$. The corresponding plots of $\operatorname{Re}\left(k_{z}\right)$ and $\operatorname{Im}\left(k_{z}\right)$ for $d=46 \mathrm{~nm}$ are also shown (gray curves or green curves online). first Brillouin zone for $\lambda=10 \mu \mathrm{m}$ with respect to different $d$ values are plotted in Fig. 1(b). Here the graphene sheets are initially assumed to be freestanding in air with $\varepsilon_{d}=1$ for simplicity. Obviously, there is a critical period below which $k_{z}$ drops dramatically at the Brillouin zone center $(\varphi=0)$. At $\varphi=0$, Eq. (2) reduces to $\tanh (\kappa d / 2)=\kappa \xi / 2$, which has only a trivial solution of $\kappa=0$ as $d<\xi$. Therefore, the critical period equals $\xi$. As shown in Fig. 1(b), when $d \gg \xi$, the wave vector of SPPs in the MGSA varies slowly around the wave vector of SPPs in an individual graphene sheet $\left(k_{z} \approx k_{\mathrm{SPP}}\right)$, displaying the weak coupling of adjacent graphene sheets in the array. Therefore, the dramatic variation of the diffraction curve in $d<\xi$ reflects the strong coupling of SPPs.

The loss of SPPs in the MGSA is characterized by the imaginary part of $k_{z}$, which is plotted in Fig. 1(c) as a function of $\varphi$ and $d$. The longitudinal propagation distance of SPPs can be simply written as $L_{z}=\left[2 \operatorname{Im}\left(k_{z}\right)\right]^{-1}$. At the Brillouin zone center, the loss is evidently lower within the strong coupling regime than in other regimes because of $\kappa \approx 0$ and hence the small $E_{z}$ field. Considering the surface power loss density $P_{\text {loss }}=\operatorname{Re}\left(\sigma_{g}\right)\left|E_{z}\right|^{2} / 2$ [24], the loss due to the conduction current in graphene remains at a low level for small $E_{z}$. However, it would increase at the Brillouin zone edges where $\kappa \gg k_{0}$ and the electric field is significantly enhanced. It is found that $L_{z}=0.78 \mu \mathrm{m}$ $\left(L_{z} / \lambda_{p}=8.8\right)$ at $d=20 \mathrm{~nm}$ and $\varphi=\pi$, while in comparison, $L_{z}=1.5 \mu \mathrm{m}$ at $\varphi=0.2 \pi$ in the vicinity of the Brillouin zone center, doubling the propagation distance of $0.74 \mu \mathrm{m}$ in monolayer graphene. When $d \gg \xi$, the even superposition of SPPs makes the fields between adjacent graphene destructive and most of the energy is carried in graphene, resulting in a larger propagation loss at the Brillouin zone center [15]. The singularity around $d=\xi$ is because of the abrupt change from a high loss SPP mode to a lossless one $(\kappa=0)$ for which the energy is carried in the dielectric. In this Letter, we only focus on the strong coupling regime $(d<\xi)$.

We consider a situation that SPPs are individually excited in each graphene sheet of the MGSA. The amplitude of SPPs in the $n$th graphene is given by $A_{n}=A_{0} \exp \left(-\frac{x_{n}^{2}}{W_{0}^{2}}\right) \exp \left(\operatorname{in} \varphi_{0}\right)$, where $x_{n}=n d$ denotes the location of graphene, $W_{0}$ is the Gaussian width of the amplitude envelope, and $\varphi_{0}$ is the initial phase difference between adjacent graphene. This initial condition could yield SPP modes in the MGSA with the Bloch momenta centered at $\varphi_{0}$. In practice, the SPPs in individual graphene sheets can be excited by nanoemitters such as quantum dots, molecules, dielectric or metallic protuberances lying on graphene [12]. The amplitude and initial phase of SPPs could be controlled by adjusting the size and $z$ direction locations of the nanoemitters, respectively. Figures 2(a)-2(d) show the simulation results of SPP intensity $\left(|\boldsymbol{E}|^{2}\right)$ distributions in the MGSA for different $\varphi_{0}$ by using the finite-difference frequency-domain (FDFD) 

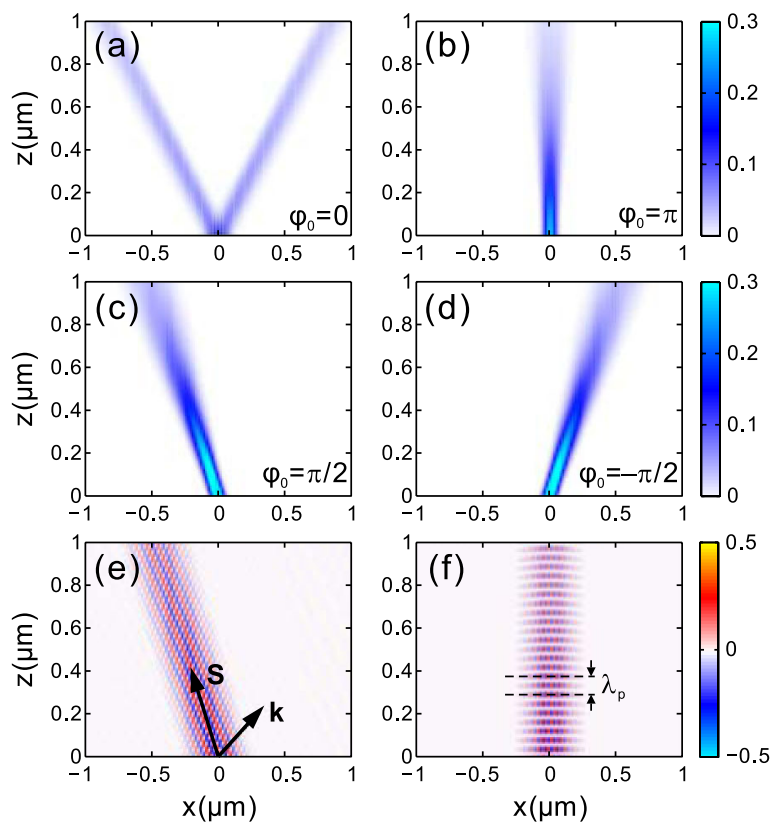

FIG. 2 (color online). (a)-(d) Intensity $\left(|\boldsymbol{E}|^{2}\right)$ distributions of SPPs in the MGSA for different excitation conditions: (a) $\varphi_{0}=0$, (b) $\varphi_{0}=\pi$, (c) $\varphi_{0}=\pi / 2$, and (d) $\varphi_{0}=-\pi / 2$, where $\varphi_{0}$ denotes the initial phase difference of SPPs between neighboring graphene. The array period is $d=20 \mathrm{~nm}$ and incident field width $W_{0}=3 d$. (e) $E_{x}$-field distribution for $\varphi_{0}=\pi / 2$. (f) $E_{x}$-field distribution for $\varphi_{0}=\pi$. In (e) and (f), the incident field has a width of $W_{0}=8 d$.

method [25]. Here we choose $d=20 \mathrm{~nm}$ and $W_{0}=3 d$. The graphene is treated as an ultrathin metallic film with a thickness of $\Delta=1 \mathrm{~nm}$ and a relative equivalent permittivity of $\varepsilon_{g \text {,eq }}=1+i \sigma_{g} \eta_{0} /\left(k_{0} \Delta\right)$ [6,7]. The minimum mesh size equals $\Delta / 5$ in the FDFD computations. The numerical convergence has been checked by using a smaller $\Delta$ of $0.5 \mathrm{~nm}$ in calculating the effective index of SPPs in a single graphene sheet. The deviation is less than $2 \%$. At $\varphi_{0}=0$, when SPPs are incident in phase, interestingly, the field splits into two separated beams propagating with a splitting angle of $79.6^{\circ}$, as shown in Fig. 2(a). Moreover, the two beams are well collimated and suffer low propagation loss. Each beam has a width of $W_{x}=143 \mathrm{~nm}(\sim \lambda / 70)$ in the $x$ direction at $z=1 \mu \mathrm{m}$. In contrast, SPPs experience stronger diffraction at $\varphi_{0}=\pi$ and transmit in a shorter distance, as shown in Fig. 2(b). The beam width becomes $W_{x}=209 \mathrm{~nm}$ at $z=1 \mu \mathrm{m}$. For $\varphi_{0}= \pm \pi / 2$, the beams tilt to the directions of $\theta=\mp 27.1^{\circ}$ with equivalent widths of $W_{x}=235 \mathrm{~nm}$ at $z=1 \mu \mathrm{m}$, as shown in Figs. 2(c) and 2(d).

Figure 2(e) shows the $E_{x}$-field distribution of SPPs in the MGSA as $\varphi_{0}=\pi / 2$. The width of the incident field has been changed to $W_{0}=8 d$ to avoid diffraction. The Poynting flux $S$ indicates the direction of energy transfer. The phase variation is determined by the wave vector $\boldsymbol{k}$, which is normal to the equiphase surfaces. It is found that $S_{x}<0$ and $k_{x}>0$ in the lateral direction, which evinces
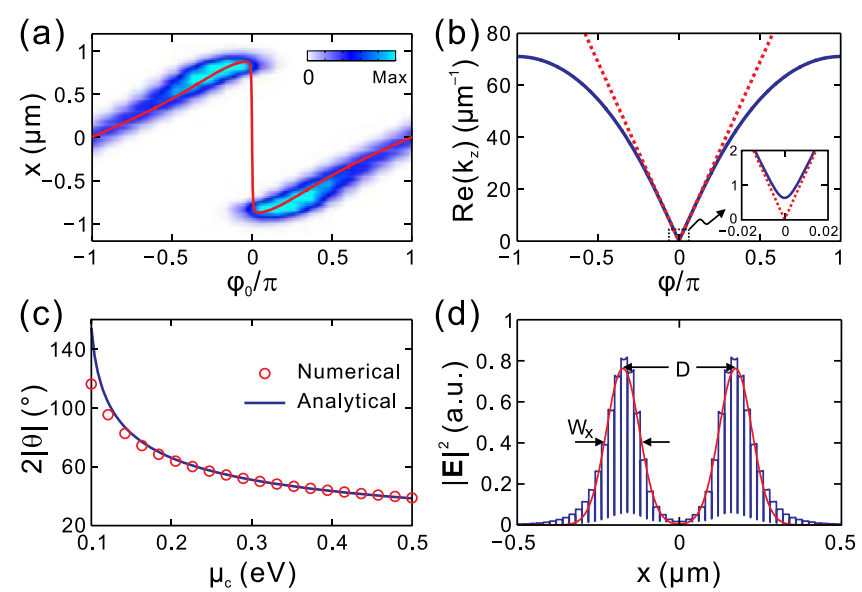

FIG. 3 (color online). (a) Numerical results of SPP intensity distribution along the $x$ direction at $z=1 \mu \mathrm{m}$ as $\varphi_{0}$ is varying. The solid curve indicates the theoretical prediction of the SPP beam positions. (b) Diffraction relation of SPPs for $d=20 \mathrm{~nm}$. The dotted lines represent the asymptotes of the diffraction curve in the vicinity of the Brillouin zone center. (c) Beam splitting angle $2|\theta|$ as a function of the chemical potential of graphene. (d) Intensity $\left(|\boldsymbol{E}|^{2}\right)$ distribution of SPPs at $z=0.5 \mu \mathrm{m}$ for $\mu_{c}=0.5 \mathrm{eV}$. The envelope of the intensity is represented by Gaussian fitting with each beam having a width of $W_{x}$.

the negative coupling in MGSAs under out-of-phase illumination. Negative coupling is discovered in the situation that the energy and phase of the mode propagate in opposite directions $\left(S_{x} k_{x}<0\right)$ when it couples from one waveguide to another [26]. Such behavior stems largely from the concave diffraction curve of MGSAs that is contrary to conventional periodic dielectric waveguide arrays where positive coupling is found [22].

Figure 2(f) shows the $E_{x}$-field distribution at $\varphi_{0}=\pi$. The modal wavelength of the collective SPPs can be written as $\lambda_{p}=2 \pi / \operatorname{Re}\left(k_{z}\right)$ [27]. At $d=20 \mathrm{~nm}$, the modal wavelength reaches $\lambda_{p}=88.4 \mathrm{~nm}$. In contrast, the wavelength of SPPs in monolayer graphene is $\lambda_{\mathrm{SPP}}=144.6 \mathrm{~nm}$. According to Eq. (2), the dispersion relation reduces to $\operatorname{coth}(\kappa d / 2)=\kappa \xi / 2$ at the Brillouin zone edges. In case $d \ll \xi$, we approximately have $\lambda_{p} \approx 2 \pi / \kappa=\pi(d \xi)^{1 / 2}$, and hence $\lambda_{p} \propto\left(\mu_{c} d / \varepsilon_{d}\right)^{1 / 2} \omega^{-1}$. In order to decrease the modal wavelength, accordingly, one may either decrease the array period or fix the chemical potential at a low level. Considering that $\lambda_{p} / \lambda_{\mathrm{SPP}}=(d / \xi)^{1 / 2}$, the modal wavelength of SPPs in the MGSA could be $\sim 3$ times smaller than that in monolayer graphene at $d=5 \mathrm{~nm}$.

The output SPP intensity profile as a function of $\varphi$ and position in the $x$ direction is illustrated in Fig. 3(a) at $z=$ $1 \mu \mathrm{m}$. The positions opposite to $\varphi_{0}$ evidently demonstrate the negative coupling of SPPs in the MGSA. The direction of the Poynting flux is determined by the group velocity and is given by $\theta\left(\varphi_{0}\right)=-\left.\arctan \left(d \partial k_{z} / \partial \varphi\right)\right|_{\varphi=\varphi_{0}}$. At a distance of $L$ in the $z$ direction, the intensity maximum of output SPPs locates at $x=L \tan (\theta)=-L d \partial k_{z} /\left.\partial \varphi\right|_{\varphi=\varphi_{0}}$. 


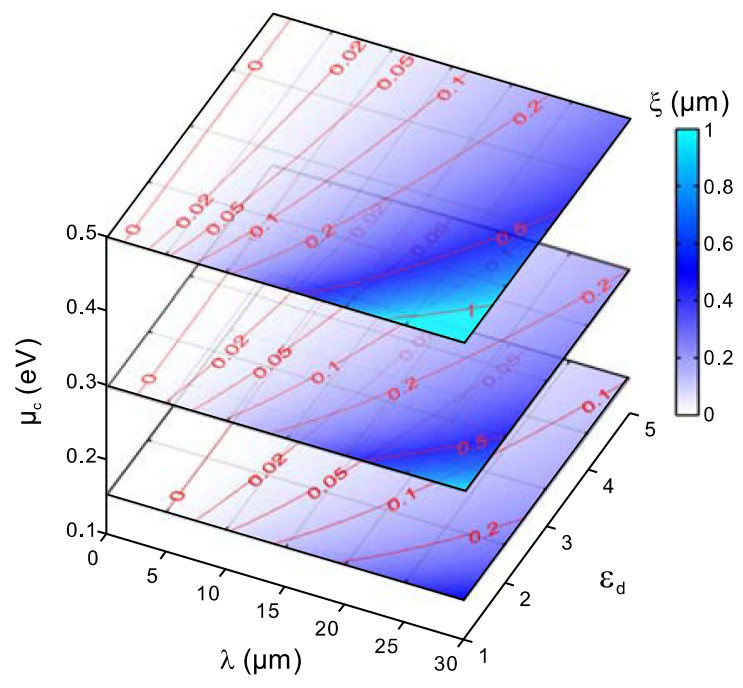

FIG. 4 (color online). Influence of the wavelength, dielectric permittivity, and chemical potential on $\xi$. The solid curves labeled with numbers depict the contour lines of $\xi$ on the $\mu_{c}$ planes of $\mu_{c}=0.15 \mathrm{eV}, 0.3 \mathrm{eV}$, and $0.5 \mathrm{eV}$.

The analytical result is plotted with a solid curve in Fig. 3(a), which coincides fairly well with the numerical data. In the vicinity of the Brillouin zone center $(|\varphi| \approx 0)$, according to Eq. (2), the diffraction relation $k_{z}(\varphi)$ can be rewritten as

$$
k_{z}^{2}-\frac{\varphi^{2}}{d(\xi-d)}=\varepsilon_{d} k_{0}^{2}
$$

When $|\varphi| \gg \varphi_{c}$ (still around the Brillouin zone center) with $\varphi_{c}=k_{0}\left[\varepsilon_{d} d(\xi-d)\right]^{1 / 2}$, the diffraction curve could be approximately represented by $k_{z}=[d(\xi-d)]^{-1 / 2}|\varphi|$ as depicted in Fig. 3(b). The linear dependence of $k_{z}$ on $|\varphi|$ indicates a diffraction-free propagation of SPPs around the Brillouin zone center except for a tiny nonlinear region $|\varphi|<\varphi_{c}$ since $\varphi_{c} / \pi=0.0046$, as shown in the inset of Fig. 3(b). Thus the uncertainty of Bloch momenta because of the limited width of input waves $\left(W_{0}=3 d\right)$ could result in two main directions for SPP propagation. The diffraction angle of SPPs is given by $\theta= \pm \arctan \left(\sqrt{\frac{d}{\xi-d}}\right)$. The theoretically predicted angle of $|\theta|=41.2^{\circ}$ agrees well with the numerical value of $39.8^{\circ}$. As the optical properties of graphene can be modulated by external static electric or magnetic fields, the beam splitting angle of SPPs in MGSAs is able to be artificially controlled. We plot in Fig. 3(c) the relationship of the splitting angle $2|\theta|$ versus the chemical potential. It can be seen that the splitting angle decreases as the chemical potential increases. The prediction agrees well with the FDFD computations. At $\mu_{c}=0.1 \mathrm{eV}$, a large splitting angle of $120^{\circ}$ can be reached. For very small $d$, we have $|\theta| \propto(d / \xi)^{1 / 2}$. The splitting angle can also be enlarged by utilizing dielectric materials of higher permittivity since $\xi$ is inversely proportional to $\varepsilon_{d}$. Figure 3(d) shows the intensity distribution across the MGSA at $z=$ $0.5 \mu \mathrm{m}$ for $\mu_{c}=0.5 \mathrm{eV}$ by using FDFD computations.
The splitting angle is obtained by Gaussian fitting of the output intensity at a distance of $L=0.5 \mu \mathrm{m}$ in the $z$ direction and $|\theta|=\arctan [D /(2 L)]$, where $D$ is the distance between the split beams. The fitted beam width $W_{x}=144 \mathrm{~nm}$.

In Fig. 4, we render the function $\xi\left(\lambda, \varepsilon_{d}\right)$ on different planes with respect to $\mu_{c}=0.15 \mathrm{eV}, 0.3 \mathrm{eV}$, and $0.5 \mathrm{eV}$. On each $\mu_{c}$ plane (vertical to the $\mu_{c}$ axis), $\xi$ increases as $\lambda$ increases and $\varepsilon_{d}$ decreases. As the chemical potential increases, the values of $\xi$ undergo a blue shift. That means higher photon energy is able to yield strong coupling in MGSA composed of higher chemical potential graphene sheets. In practice, graphene is usually supported by dielectric materials such as silicon dioxide or boron nitride. For example, as $\varepsilon_{d}=4$ for silicon dioxide, in order to obtain strong coupling, the wavelength should be $\lambda>12.7 \mu \mathrm{m}$ at $d=20 \mathrm{~nm}$ and $\mu_{c}=0.15 \mathrm{eV}$. Alternatively, we should increase the chemical potential to $\mu_{c}>0.23 \mathrm{eV}$ when the wavelength is fixed at $\lambda=10 \mu \mathrm{m}$.

It is worth comparing SPPs in metal thin layers with that in graphene. For a single layer of metal, there should be a symmetric mode (long range SPPs) and an antisymmetric mode (short range SPPs) as the metal thickness is very small $[28,29]$. The symmetric mode has a much longer propagation length than SPPs in graphene [30]. Nevertheless, the antisymmetric mode is more like SPPs in graphene due to the tight confinement of field but with large propagation loss. Most of previous works [16-18] on arrays of metal layers concerned the weak coupling of SPPs. As the metal thickness decreases, according to the diffraction relation [18], similar phenomena such as beam splitting discovered in graphene may occur in arrays of metal layers in the nearinfrared range but only for the metals with a thickness of several nanometers that are nearly two-dimensional materials. Such thin metal films tend to be granularlike with discontinuities and significant roughness in the practical case $[31,32]$. Moreover, the loss of near-infrared SPPs in arrays of metal layers such as silver thin films is much larger than that of far-infrared SPPs in MGSAs when both have the modal wavelength at the same level. The low loss and tunability render graphene arrays big advantages over the arrays of thin metal layers.

In conclusion, we have analyzed the coupling between SPPs in a discrete optical system made of MGSAs. New phenomena have been revealed analytically and demonstrated with full-vectorial numerical computations. Strong coupling between SPPs emerges when the graphene sheets are arranged tightly with a period smaller than a critical value. Within this regime and for out-of-phase illumination, these structures display negative coupling between the individual SPPs of each monolayer; i.e., the diffraction angle of the resulting beam is always opposite to its Bloch momentum. However, for in-phase illumination, the incident beam splits into two separate beams that display nearly diffraction-free propagation and low loss. The fascinating 
properties of graphene make it a good platform to investigate the wave coupling behaviors in discrete optical systems. We believe that our fundamental study could find promising applications for graphene in optoelectronic devices and circuits.

The work is partially supported by the Agency for Science, Technology, and Research (A*STAR) under Grant Nos. 0921450030, 0921540099, 0921540098 and the Ministry of Science and Technology of China under Grant No. 2009DFA52300 for China-Singapore collaborations. F. J. G.-V. acknowledges financial support from the European Research Council under Grant No. 290981 (PLASMONANOQUANTA).

*wangb@imre.a-star.edu.sg

†h-teng@imre.a-star.edu.sg

[1] K. Novoselov, A. Geim, S. Morozov, D. Jiang, Y. Zhang, S. Dubonos, I. Grigorieva, and A. Firsov, Science 306, 666 (2004).

[2] S. K. Pati, T. Enoki, and C. N. R. Rao, Graphene and Its Fascinating Attributes (World Scientific, Singapore, 2011).

[3] F. Bonaccorso, Z. Sun, T. Hasan, and A. Ferrari, Nature Photon. 4, 611 (2010).

[4] A. K. Geim, Science 324, 1530 (2009).

[5] G. W. Hanson, J. Appl. Phys. 103, 064302 (2008).

[6] J. Christensen, A. Manjavacas, S. Thongrattanasiri, F. H. L. Koppens, and F. J. Garcia de Abajo, ACS Nano 6, 431 (2012).

[7] A. Vakil and N. Engheta, Science 332, 1291 (2011).

[8] G. W. Hanson, J. Appl. Phys. 104, 084314 (2008).

[9] W. Zhou, J. Lee, J. Nanda, S. T. Pantelides, S. J. Pennycook, and J.-C. Idrobo, Nature Nanotech. 7, 161 (2012).

[10] A. Y. Nikitin, F. Guinea, F. J. García-Vidal, and L. Martín-Moreno, Phys. Rev. B 84, 161407(R) (2011).

[11] F. H. L. Koppens, D. E. Chang, and F. J. García de Abajo, Nano Lett. 11, 3370 (2011).

[12] A. Y. Nikitin, F. Guinea, F. J. García-Vidal, and L. MartínMoreno, Phys. Rev. B 84, 195446 (2011).

[13] J. Chen, M. Badioli, P. Alonso-González, S. Thongrattanasiri, F. Huth, J. Osmond, M. Spasenović,
A. Centeno, A. Pesquera, P. Godignon, A. Z. Elorza, N. Camara, F. J. García de Abajo, R. Hillenbrand, and F. H. L. Koppens, Nature (London) 487, 77 (2012).

[14] Z. Fei, A. S. Rodin, G. O. Andreev, W. Bao, A. S. McLeod, M. Wagner, L. M. Zhang, Z. Zhao, M. Thiemens, G. Dominguez, M. M. Fogler, A. H.C. Neto, C. N. Lau, F. Keilmann, and D. N. Basov, Nature (London) 487, 82 (2012).

[15] B. Wang, X. Zhang, X. Yuan, and J. Teng, Appl. Phys. Lett. 100, 131111 (2012).

[16] E. Verhagen, R. De Waele, L. Kuipers, and A. Polman, Phys. Rev. Lett. 105, 223901 (2010).

[17] X. Fan, G. P. Wang, J.C. W. Lee, and C. T. Chan, Phys. Rev. Lett. 97, 073901 (2006).

[18] L. Verslegers, P. B. Catrysse, Z. Yu, and S. Fan, Phys. Rev. Lett. 103, 033902 (2009).

[19] V. Gusynin, S. Sharapov, and J. Carbotte, J. Phys. Condens. Matter 19, 026222 (2007).

[20] P. Y. Chen and A. Alù, ACS Nano 5, 5855 (2011).

[21] K. I. Bolotin, K. Sikes, Z. Jiang, M. Klima, G. Fudenberg, J. Hone, P. Kim, and H. Stormer, Solid State Commun. 146, 351 (2008).

[22] T. Pertsch, T. Zentgraf, U. Peschel, A. Bräuer, and F. Lederer, Phys. Rev. Lett. 88, 093901 (2002).

[23] D. N. Christodoulides, F. Lederer, and Y. Silberberg, Nature (London) 424, 817 (2003).

[24] J.D. Jackson, Classical Electrodynamics (Wiley, New York, 1998), 3rd ed.

[25] M.L. Brongersma and P.G. Kik, Surface Plasmon Nanophotonics (Springer, New York, 2007).

[26] N. K. Efremidis, P. Zhang, Z. Chen, D. N. Christodoulides, C.E. Rüter, and D. Kip, Phys. Rev. A 81, 053817 (2010).

[27] Y. Liu, G. Bartal, D. A. Genov, and X. Zhang, Phys. Rev. Lett. 99, 153901 (2007).

[28] E. N. Economou, Phys. Rev. 182, 539 (1969).

[29] P. Berini, Adv. Opt. Photon. 1, 484 (2009).

[30] P. Tassin, T. Koschny, M. Kafesaki, and C. M. Soukoulis, Nature Photon. 6, 259 (2012).

[31] H. Liu, B. Wang, L. Ke, J. Deng, C. C. Chum, S. L. Teo, L. Shen, S. A. Maier, and J. Teng, Nano Lett. 12, 1549 (2012).

[32] P. Nagpal, N.C. Lindquist, S.H. Oh, and D. J. Norris, Science 325, 594 (2009). 